\title{
Identification of Estrogen Receptor $\alpha$ Antagonists from Natural Products via In Vitro and In Silico Approaches
}

\author{
Xiaocong Pang, ${ }^{1}$ Weiqi Fu, ${ }^{1}$ Jinhua Wang, ${ }^{1,2,3}$ De Kang, ${ }^{1}$ Lvjie $\mathrm{Xu},{ }^{1}$ Ying $\mathrm{Zhao},{ }^{1}$ \\ Ai-Lin Liu ${ }^{1},{ }^{1,2,3}$ and Guan-Hua Du (iD) $1,2,3$ \\ ${ }^{1}$ Institute of Materia Medica, Chinese Academy of Medical Sciences and Peking Union Medical College, Xian Nong Tan Street, \\ Beijing 100050, China \\ ${ }^{2}$ Beijing Key Laboratory of Drug Target Research and Drug Screening, Chinese Academy of Medical Sciences and Peking Union \\ Medical College, Beijing 100050, China \\ ${ }^{3}$ State Key Laboratory of Bioactive Substance and Function of Natural Medicines, Chinese Academy of Medical Sciences and Peking \\ Union Medical College, Beijing 100050, China
}

Correspondence should be addressed to Ai-Lin Liu; liuailin@imm.ac.cn and Guan-Hua Du; dugh@imm.ac.cn

Xiaocong Pang and Weiqi Fu contributed equally to this work.

Received 15 February 2018; Revised 1 April 2018; Accepted 11 April 2018; Published 10 May 2018

Academic Editor: Valentina Pallottini

Copyright (c) 2018 Xiaocong Pang et al. This is an open access article distributed under the Creative Commons Attribution License, which permits unrestricted use, distribution, and reproduction in any medium, provided the original work is properly cited.

\begin{abstract}
Estrogen receptor $\alpha(\mathrm{ER} \alpha)$ is a successful target for ER-positive breast cancer and also reported to be relevant in many other diseases. Selective estrogen receptor modulators (SERMs) make a good therapeutic effect in clinic. Because of the drug resistance and side effects of current SERMs, the discovery of new SERMs is given more and more attention. Virtual screening is a validated method to high effectively to identify novel bioactive small molecules. Ligand-based machine learning methods and structure-based molecular docking were first performed for identification of ER $\alpha$ antagonist from in-house natural product library. Naive Bayesian and recursive partitioning models with two kinds of descriptors were built and validated based on training set, test set, and external test set and then were utilized for distinction of active and inactive compounds. Totally, 162 compounds were predicted as ER antagonists and were further evaluated by molecular docking. According to docking score, we selected 8 representative compounds for both ER $\alpha$ competitor assay and luciferase reporter gene assay. Genistein, daidzein, phloretin, ellagic acid, ursolic acid, (-)-epigallocatechin-3-gallate, kaempferol, and naringenin exhibited different levels for antagonistic activity against ER $\alpha$. These studies validated the feasibility of machine learning methods for predicting bioactivities of ligands and provided better insight into the natural products acting as estrogen receptor modulator, which are important lead compounds for future new drug design.
\end{abstract}

\section{Introduction}

Estrogens are the prime female sex hormones and play vital roles for menstrual cycle regulation and female sexual development [1]. However, in the past twenty years, it was reported that estrogens affect both males and females physiologically, including the metabolism of carbohydrate and lipid, the skeletal homeostasis, the cardiovascular system, and the central nervous system (CNS) $[2,3]$. Extensive evi- dence suggests that estrogens attenuate oxidative stress by preventing generation of reactive oxygen species (ROS), which was related either to regulation of ROS-generating enzymes or augmentation of ROS-eliminating mechanisms [4]. The biological effects of estrogen are regulated through estrogen receptors [5]. ER $\alpha$ (estrogen receptor $\alpha$ ) has a wide distribution in the development and functioning of various organs and tissues in the body, such as the brain, bone, urogenital tract, and cardiovascular system $[6,7]$. ER $\alpha$-positive 
and estrogen-dependent breast cancers make up a high proportion (more than 70\%) [8]. Endocrine therapy is considered as an effective treatment through blocking the ER transcription. It was also reported that $\operatorname{ER} \alpha$ is clinically relevant in endometrial, ovarian, and other cancer types $[9,10]$. Therefore, ER $\alpha$ was an ideal pharmaceutical target and a lot of $\operatorname{ER} \alpha$ ligands have been successfully developed for $\mathrm{ER} \alpha$ positive breast cancer treatment $[11,12]$.

Selective estrogen receptor modulators (SERMs) have special action mode with ER, which act as antagonists for antibreast cancer in breast tissue, but agonists in other tissues such as the bone and cardiovascular system [13]. Therefore, SERMs, such as tamoxifen, prevent bone density loss and also benefit the cardiovascular system [14]. Although there are lots of advantages of SERMs, they still remain deficiencies. For instance, tamoxifen for long-term treatment leads to the development of endometrial cancer and drug resistance $[15,16]$. Therefore, new SERMs with higher activity and fewer side effects are given more and more attention [17].

Natural products have a wide molecular diversity and range of biological properties to provide a primary resource for high-throughput screening (HTS) and virtual screening [18]. There are a lot of natural product databases for collecting constitutes of herbs, such as TCM Database@Taiwan [19], TCMSP [20], TCMID [21], CEMTDD [22], SuperToxic [23], and SuperNatural [24], providing much data for screening and mechanism of action studies [25]. It was reported that some flavonoids derived from herbs were capable of reducing bone loss and bone deterioration associated with estrogen deficiency, but they could not lead to uterotrophic effects [26]. Therefore, natural products could be potential SERMs for the treatment of cancers or other diseases.

The discovery of leads by HTS is high cost and timeconsuming and demands large for labor. Therefore, virtual screening that needs less time and investment has been widely used for facilitating drug discovery [27]. Ligandbased drug design (LBDD) and structure-based drug design (SBDD) techniques were two powerful approaches to find or develop a hit or lead compound as drug candidate [28]. LBDD refers to pharmacophore, quantitative structure-activity relationship (QSAR), and machine learning techniques. SBDD includes molecular docking, molecular dynamics, and pharmacophore based on protein structure [29]. Therefore, based on the availability of the known ligands and/or target structure information, we should apply proper method to build virtual screening model. In addition, integrating the different methods together is beneficial for making up their deficiency and improving the reliability [30].

In our study, we attempted to combine the machine learning methods and molecular docking for identifying novel $\mathrm{ER} \alpha$ ligands from in-house natural product database. Naive Bayesian (NB) and recursive partitioning (RP) models were built and validated based on training set and test set and then were utilized for classification of active and inactive from the database. These compounds predicted as ER antagonists were further evaluated by molecular docking.
According to docking score and the representative structures, several compounds were selected for $\mathrm{ER} \alpha$ competitor assay and luciferase reporter gene assay for their antagonistic activity against $\mathrm{ER} \alpha$. These studies provided better insight into the natural products acting as estrogen receptor modulators, which were important lead compounds for rational design of new SERMs in the future.

\section{Materials and Methods}

2.1. Data Collection and Preparation. There were two datasets prepared. After eliminating the duplicate structures, $\mathrm{ER} \alpha$ antagonists with the values of $\mathrm{IC}_{50}$ less than $10 \mu \mathrm{M}$ were obtained from the BindingDB database [31]. In addition, corresponding decoy datasets were generated in DUD-E online database [32] with the above ER $\alpha$ antagonists. The training set and test set were generated randomly. Then inorganic salt atoms of compounds were deleted, and subsequently, the compounds were added hydrogen atoms, deprotonated strong acids, protonated strong bases, built valid three-dimensional conformation, and minimized of energy by Molecular Operating Environment (MOE). All ER $\alpha$ antagonists and decoys were marked with "1" and "-1," respectively.

2.2. Molecular Descriptors. The MOE software is able to compute 186 2D descriptors as well as 148 3D molecular descriptors [33]. 2D molecular descriptors are defined to be numerical properties that can be calculated from the connection table representation of a molecule. $2 \mathrm{D}$ descriptors refer to notation and terminology, physical properties, subdivided surface areas, Kier \& Hall connectivity and kappa shape indices, adjacency and distance matrix descriptors, pharmacophore feature descriptors, and partial charge descriptors. 3D molecular descriptors consist of potential energy descriptors, MOPAC descriptors, surface area, volume and shape descriptors, and conformationdependent charge descriptors. Similarly, Discovery Studio 2016 (DS) was used to calculate the 2D descriptors, which were made up of AlogP, estate keys, molecular properties, molecular property counts, surface area and volume, and topological descriptors. Extended-connectivity fingerprint6 (ECFP-6) was also calculated with this software.

2.3. Molecular Descriptor Selection. To avoid the complexity and increase the efficiency of models, we firstly selected the proper molecular descriptor by Pearson correlation analysis and stepwise variable selection method [34]. Pearson correlation analysis was used to delete the descriptors not remarkably associated with activity and highly associated with each other. The criterion of elimination was that descriptors with correlation coefficients with less than 0.1 were removed. In addition, when correlation coefficient between two descriptors was more than 0.9 , the descriptor with a lower correlation coefficient to activity would be deleted. Then, the rest of the descriptors were selected by stepwise analysis. The initial regression equation was created by the first descriptor. Then, other descriptors were imported to the equation in tune. At the same time, every 
new regression equation would be subjected to a significance test for evaluating the addition of a new descriptor. For example, the new descriptor would be removed, if the regression equation was not "statistically significant." In addition, the descriptors were also deleted when they did not conform to "statistically significant" in the equation. The process would be completed if there were no descriptors imported or deleted.

\subsection{Machine Learning Models}

2.4.1. Naive Bayesian (NB) Classifier. Based on Bayes' theorem, Bayesian categorization model is a useful probabilistic classification model [35]. During a learning process, the algorithm could generate a series of Boolean features according to the input descriptors. The frequency of occurrence of each feature in the good subset was calculated in all data samples. Then, features of the sample were generated for applying the model to a particular sample, and weights for each feature were calculated through Laplacian-adjusted probability estimate, which was a relative predictor of the possibility of that sample being from the good subset. Bayesian categorization can process a great quantity of data with high efficiency and is immune to random noise. In this study, NB classifiers were carried out by DS 2016. The parameters remained their default values.

2.4.2. Recursive Partitioning (RP) Classifier. RP generates decision tree to reveal the relationship between a dependent property (activity) and a set of independent properties (molecular descriptors) [36]. The input data were divided into two subsets based on a particular molecular descriptor and corresponding splitting value at each node of the decision tree. When there were no more significant nodes, the splitting process was finished. RP classifiers were established by using Discovery Studio (DS) 2016. In RP model, to avoid excessive partitioning, the minimum number of samples per node was set as 10 and the maximum tree depth was used as 20. Each class was weighted equally. The class for a node is the class with the greatest weighted sum of samples in the node. The Gini index was used as a measure of the increase in node purity as the result of a split.

2.4.3. Model Performance. NB and RP classifiers with the two kinds of descriptors, and ECFP_6 was initially generated. Subsequently, 5-fold cross-validation for the training set, test set, and external test set was used to evaluate the performance of NB. Y-scrambling was also employed to prevent $\mathrm{NB}$ and $\mathrm{RP}$ performance from a result of chance correlation for the best models. Performances of NB and RP models were evaluated by calculating the true positives (TP), true negatives (TN), false negatives (FN), false positives (FP), sensitivity (SE), and specificity (SP), prediction accuracy of antagonist $\left(\mathrm{Q}^{+}\right)$, prediction accuracy of nonantagonists $\left(\mathrm{Q}^{-}\right)$, and Matthews correlation coefficient (MCC) [33].

$$
\begin{aligned}
\mathrm{SE} & =\frac{\mathrm{TP}}{\mathrm{TP}+\mathrm{FN}}, \\
\mathrm{SP} & =\frac{\mathrm{TN}}{\mathrm{TN}+\mathrm{FP}}, \\
Q^{+} & =\frac{\mathrm{TP}}{\mathrm{TP}+\mathrm{FP}}, \\
Q^{-} & =\frac{\mathrm{TN}}{\mathrm{TN}+\mathrm{FN}}, \\
\mathrm{MCC} & =\frac{(\mathrm{TP} \times \mathrm{TN})-(\mathrm{FN} \times \mathrm{FP})}{\sqrt{(\mathrm{TP}+\mathrm{FN})(\mathrm{TP}+\mathrm{FP})(\mathrm{TN}+\mathrm{FN})(\mathrm{TN}+\mathrm{FP})}} .
\end{aligned}
$$

2.5. Molecular Docking. Molecular docking was investigated to further study the binding mode of $\mathrm{ER} \alpha$ and compounds predicted by NB and RP classifiers. We utilized the LibDock and CDOCKER protocol of DS 2016 for docking analysis. The crystal structure of $\mathrm{ER} \alpha$ was obtained from the Protein Data Bank (PDB ID: 3ERT) [37]. LibDock is a useful algorithm for docking small molecules into an active receptor pocket. Primarily, a hotspot map is generated for the receptor active site which contains polar and apolar groups. This hotspot map is subsequently utilized to form favorable interactions by strictly aligning the ligand conformations. The ligand poses with top scoring are saved after a final energyminimization step [38]. CDOCKER is the other important docking program in DS using a rigid receptor and CHARMmfield [39]. The interaction energy for each final pose of ligands with CHARMm energy was calculated, and the top scoring (most negative, thus favorable to binding) poses are retained. The structure of $\operatorname{ER} \alpha$ firstly was prepared through removing water, adding hydrogen, and then we used clean protein module in DS to correct problems, such as nonstandard naming, protein residue connectivity, missing sidechain, or backbone atoms. The compounds also were prepared by hydrogen addition, conversion into 3D structures, $\mathrm{pH}$-based ionization, and charge neutralization [40]. The original ligand, 4-hydroxytamoxifen, was selected to define the active pocket of ER $\alpha$. Then, redocking was performed to calculate the root-mean-square deviation (RMSD) values between the docking and initial poses for validating the reliability of docking methods.

2.6. ER $\alpha$ Competitor Assay. The $\mathrm{ER} \alpha$ binding affinities of representative compounds predicted as $\operatorname{ER} \alpha$ antagonists were measured by fluorescence polarization procedure using green PolarScreen $^{\mathrm{TM}}$ ER $\alpha$ Competitor Assay kit (Life Technologies, CA, United States of America) [41]. Briefly, $75 \mathrm{nM} \mathrm{ER} \alpha$ together with $4.5 \mathrm{nM}$ fluormone was mixed with a series of concentrations of the test compounds in the assay buffer. Then, they were incubated for $2 \mathrm{~h}$ in room temperature in black low volume 384-well assay plate with NBS surface (Corning, NY, United States of America) for fluorescence polarization assay. Subsequently, the detection wavelength was set at excitation wavelength $485 \mathrm{~nm}$ and emission wavelength $535 \mathrm{~nm}$ with bandwidths of 25/20 nm in EnVision Workstation version 1.7 (PerkinElmer, MA, United States 
TABLe 1: 56 molecular descriptors selected by the Pearson correlation analysis and stepwise regression.

\begin{tabular}{lcc}
\hline $\begin{array}{l}\text { Descriptor } \\
\text { class }\end{array}$ & $\begin{array}{c}\text { Numbers of } \\
\text { descriptors }\end{array}$ & Descriptors \\
& & PEOE_VSA_FNEG,PEOE_RPC+,a_base,a_ICM,a_nBr,a_nCl, \\
DS & a_nN,a_nO,a_nS,ast_violation,b_rotR,BCUT_SMR_0,BCUT_- \\
descriptors & 24 & SM_1,chi1_C,chiral_u,density,GCUT_PEOE_1,GCUT_SLOGP_1, \\
& GCUT_SMR_0,PEOE_VSA_NEG,PEOE_VSA_POS, \\
& PEOE_VSA+3,PEOE_VSA-0,PEOE_VSA-1,PEOE_VSA-3,PEOE_VSA-6, \\
& radius,reactive,rings,SMR_VSA0,vdw_vol,vsa_don
\end{tabular}

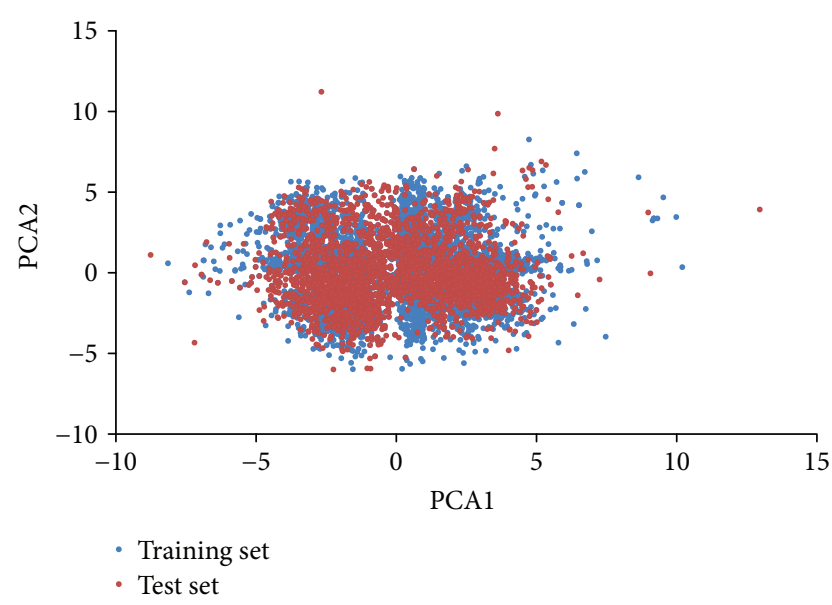

Figure 1: Diversity distribution of the training set and test set as described by principal component analysis (PCA).

of America). 2104 EnVision ${ }^{\circledR}$ Multilabel Plate Reader was performed for the measurements.

2.7. Luciferase Reporter Gene Assay for ER $\alpha$. Cell transfection and luciferase activity assay were utilized to investigate the effects of the compounds on ER transcriptional activation [42]. The human breast cancer cell line MCF-7 was obtained from the Beijing Key Laboratory of Drug Target Research and Drug Screening, Chinese Academy of Medical Sciences (Beijing, China). Dulbecco's Modified Eagle's Medium (DMEM) culture medium containing 10\% fetal calf serum (FBS), $100 \mathrm{U} / \mathrm{mL}$ penicillin, and $100 \mu \mathrm{g} / \mathrm{mL}$ streptomycin was used as complete culture medium. The culture conditions were at $37^{\circ} \mathrm{C}$, saturated humidity, and $5 \% \mathrm{CO}_{2}$. The culture medium was changed every day and when the confluence of cells reached 90\%, MCF-7 cells were digested with $0.25 \%$ trypsin containing $0.02 \%$ EDTA and then cultured under the same culture conditions. MCF-7 cells in logarithmic growth phase were resuspended in $3 \mathrm{~mL}$ complete culture medium and were seeded on 24-well plates with a density of $1 \times 10^{5}$ /well overnight. After incubation for $24 \mathrm{~h}$, cells were grown to approximately $60-80 \%$ confluence and then washed by culture medium without serum. MCF-7 cells
TABle 2: Performance of Bayesian and recursive partitioning models and their 5 -fold cross-validation results.

\begin{tabular}{lccccccccc}
\hline Model & TP & FN & FP & TN & SE & SP & MCC & $\mathrm{Q}^{+}$ & $\mathrm{Q}^{-}$ \\
\hline NB-a & 1091 & 35 & 223 & 5206 & 0.969 & 0.959 & 0.874 & 0.830 & 0.993 \\
NB-b & 1119 & 8 & 21 & 5408 & 0.993 & 0.996 & 0.985 & 0.982 & 0.999 \\
NB-c & 749 & 316 & 455 & 5037 & 0.704 & 0.917 & 0.591 & 0.622 & 0.941 \\
NB-d & 1054 & 11 & 75 & 5416 & 0.990 & 0.986 & 0.953 & 0.933 & 0.998 \\
RP-a & 1113 & 13 & 51 & 5379 & 0.988 & 0.991 & 0.966 & 0.956 & 0.998 \\
RP-b & 1111 & 15 & 49 & 5381 & 0.987 & 0.991 & 0.966 & 0.958 & 0.997 \\
RP-c & 1007 & 57 & 177 & 5314 & 0.946 & 0.968 & 0.876 & 0.850 & 0.989 \\
RP-d & 1022 & 42 & 95 & 5396 & 0.960 & 0.983 & 0.925 & 0.915 & 0.992
\end{tabular}

Note: NB-a: NB model with MOE 2D descriptors; NB-b: NB model with MOE 2D descriptors + ECFP_6; NB-c: NB model with DS 2D descriptors; NB-d: NB model with DS 2D descriptors + ECFP_6; RP-a: RP model with MOE 2D descriptors; RP-b: RP model with MOE 2D descriptors + ECFP_6; RP-c: RP model with DS 2D descriptors; RP-d: RP model with DS 2D descriptors + ECFP_6.

were cotransfected with the reporter plasmid pGL2-ERE3luc. Based on the manufacturer's instructions, transfection was mediated by lipofectamine 3000 (Invitrogen). After incubation for $12 \mathrm{~h}$, transfection medium was eliminated, and MCF-7 cells were treated with three concentrations of compounds for $24 \mathrm{~h}$. Then, MCF-7 cells were washed with phosphate-buffered saline (PBS) and cell lysis was collected after oscillation with a low speed. Finally, the luciferase activity was determined by dual-luciferase reporter assay system (Promega) according to the product manual.

\section{Results and Discussion}

3.1. Chemical Space Analysis. A total of $2075 \mathrm{ER} \alpha$ antagonists with the values of $\mathrm{IC}_{50}$ less than $10 \mu \mathrm{M}$ were collected from the BindingDB database. 7000 decoy compounds were obtained from DUD-E online database. After random assignment, the training set was generated with 1556 active and 5000 inactive compounds, and the test set was made up of 519 active and 2000 inactive compounds.

The chemical space of the training set (compounds) and test set (2519 compounds) was investigated using principal component analysis (PCA). After Pearson correlation 


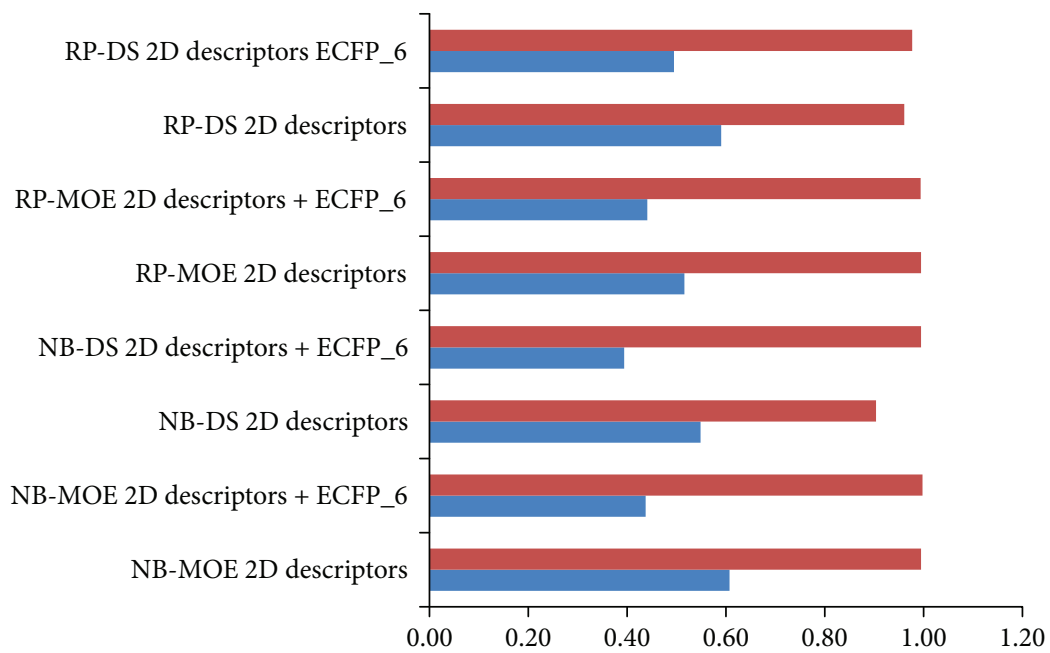

FIGURE 2: Y-scrambling for 30 times for evaluating the chance correlation possibility of naive Bayesian (NB) and recursive partitioning (RP) models by calculating Matthews correlation coefficient (MCC). The red bar represented the performance of NB and RP without Yscrambling. The blue bar showed that the MCC values decreased after Y-scrambling.

analysis and stepwise regression, we obtained 56 molecular descriptors (24 from Discovery Studio (DS) and 32 from MOE) (Table 1), which were used as the input variables for PCA. Chemical space analyzed by PCA was shown in Figure 1. It demonstrated that chemical space distributions were dispersive for all compounds, and most of the compounds in the test set are well within the chemical space of the training set.

3.2. Performance of NB and RP Models. In this study, all the classification models were initially built using RP and NB classifiers with MOE and DS 2D molecular descriptors and ECFP-6. Considering the limitation of the descriptors calculated in MOE for characterizing the important substructures or molecular fragments, molecular fingerprints (ECFP-6), together with property descriptors, were used simultaneously to establish novel prediction models. Subsequently, 5-fold cross-validations for the models were performed (Table 2). The sensitivity (SE) and prediction accuracy of antagonist $\left(\mathrm{Q}^{+}\right)$of NB classifiers with DS 2D molecular descriptors were not favorable, but added ECFP_6, the performance was excellent with SE of 0.990 and 0.933 . Y-scrambling for 30 times was as well as used to evaluate the chance correlation possibility. When the activities of compounds from the training set were disturbed, Matthews correlation coefficient (MCC) was significantly decreased, especially for NB and RP with MOE 2D descriptors in the existence of ECFP_6 (Figure 2). As shown in Table 3, DS 2D descriptors without ECFP_6 were also not better than MOE 2D descriptors in test set, which maybe DS 2D descriptors did not characterize the important substructures and molecular fragments which are critical for ER $\alpha$ antagonist. Then, compounds in external test set, which were not involved in the training and test set, were extracted from literatures published in recent years for further validation $[6,43-46]$. The external test set included 20 antagonists and 50 inactive compounds. Figure 3 suggested that NB and RP MOE 2D descriptors with ECFP_6 were the most powerful models for prediction of ER $\alpha$ antagonist.
TABLE 3: Performance of Bayesian and recursive partitioning models on the test set.

\begin{tabular}{lccccccccc}
\hline Model & TP & FN & FP & TN & SE & SP & MCC & $\mathrm{Q}^{+}$ & $\mathrm{Q}^{-}$ \\
\hline NB-a & 426 & 7 & 68 & 2018 & 0.985 & 0.967 & 0.904 & 0.862 & 0.997 \\
NB-b & 425 & 8 & 27 & 2059 & 0.981 & 0.987 & 0.952 & 0.941 & 0.996 \\
NB-c & 346 & 62 & 356 & 1756 & 0.849 & 0.832 & 0.559 & 0.493 & 0.966 \\
NB-d & 389 & 19 & 40 & 2071 & 0.954 & 0.981 & 0.917 & 0.907 & 0.991 \\
RP-a & 426 & 8 & 31 & 2055 & 0.983 & 0.985 & 0.948 & 0.932 & 0.996 \\
RP-b & 427 & 6 & 29 & 2057 & 0.987 & 0.986 & 0.953 & 0.936 & 0.997 \\
RP-c & 379 & 54 & 81 & 2005 & 0.875 & 0.961 & 0.817 & 0.824 & 0.974 \\
RP-d & 401 & 33 & 35 & 2051 & 0.925 & 0.983 & 0.906 & 0.920 & 0.984 \\
\hline
\end{tabular}

Note: NB-a: NB model with MOE 2D descriptors; NB-b: NB model with MOE 2D descriptors + ECFP_6; NB-c: NB model with DS 2D descriptors; NB-d: NB model with DS 2D descriptors + ECFP_6; RP-a: RP model with MOE 2D descriptors; RP-b: RP model with MOE 2D descriptors + ECFP_6; RP-c: RP model with DS 2D descriptors; RP-d: RP model with DS 2D descriptors + ECFP_6.

Taken together, both NB and RP models were applied for the screening of natural product database with MOE 2D descriptors and ECFP_6.

3.3. Good and Bad Fragments Given by Naive Bayesian Model. ECFP_ 6, as the structural fingerprint used in Bayesian classifier, could identify key fragments or fingerprint features frequently found in two classifying groups, which provided important information for the design of ER $\alpha$ antagonist. The top 10 favorable and 10 unfavorable fragments for $\mathrm{ER} \alpha$ binding were ranked by the Bayesian scores of the NB-b model (Figure 4). The positive fragments for ER $\alpha$ binding mainly included phenolic hydroxyl and saturated nitrogen atom. By analyzing 4-hydroxytamoxifen in the ligandbinding domain in ER $\alpha$ (PDB ID: 3ERT), we found phenolic hydroxyl could interact with Arg394 and Glu353 by forming stable hydrogen bonds. However, most fragments referring to nitrogen atoms with positive charges were observed in 


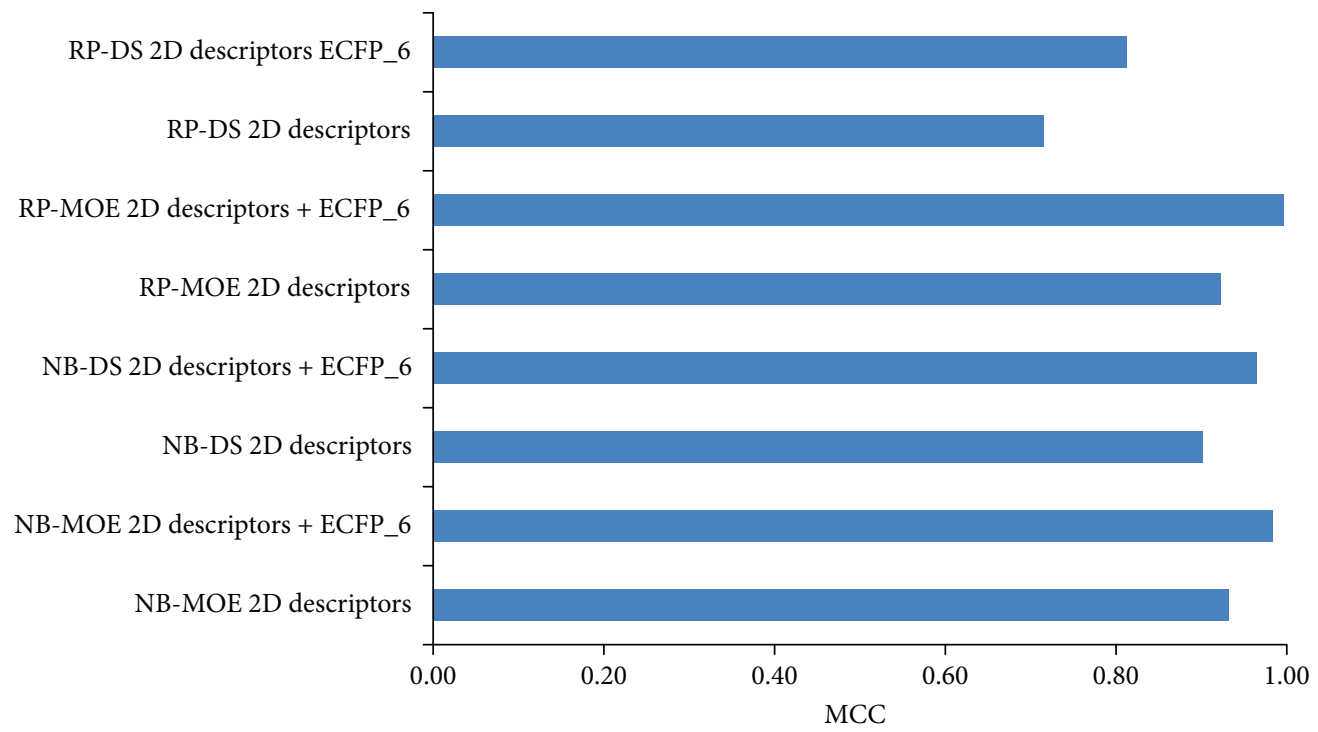

FIGURE 3: The performance of MCC value made by 8 classifiers on external test set. NB and RP MOE 2D descriptors with ECFP_6 were the most powerful models for prediction of $\mathrm{ER} \alpha$ antagonist.

the negative contributions to $\operatorname{ER} \alpha$ binding. In addition, most of unfavorable fragments contain sulfur atoms, indicating its common occurrence in many inactive ligands.

3.4. Virtual Screening of an In-House Natural Product Database for $E R \alpha$. The best models (NB-b and RP-b) were applied for virtual screening of an in-house natural product database (including 13166 compounds) to identify for ER $\alpha$ lead ligands. First of all, each compound was prepared by calculating 25 descriptors (24 DS descriptors and ECFP_6) and 33 descriptors (32 DS descriptors and ECFP_6), respectively, then NB-b and RP-b were performed to evaluate the probability as ER $\alpha$ antagonists for each compound. 393 compounds were predicted as potential ER $\alpha$ antagonists by the NB-b model, while 193 compounds were predicted as active compounds against ER $\alpha$ using the RP-b model. By analyzing the overlapping part, 162 compounds were predicted as ER $\alpha$ antagonists with the two models simultaneously. Then, these compounds were further evaluated by molecular docking. The greatest advantage of LibDock is its high speed and parallel operation, which makes it suitable for large scale applications. Therefore, we used LibDock for further screening. Then, RMSD value calculated through redocking between the docking and initial poses was $1.410 \AA$, which suggested the reliability of LibDock methods. CDOCKER uses simulated annealing to optimize each conformation in the active site region of the acceptor, thereby making docking results more accurate. Therefore, we utilized CDOCKER to analyze the interaction of ligand and receptor. We also calculate the RMSD for CDOCKER, and the value was $1.242 \AA$, which validated that the docking models could be used for further docking studies. After preparation of 162 compounds, LibDock was firstly performed for quick screening. Most of these compounds could dock to the pocket of $\mathrm{ER} \alpha$ with a wide range of LibDockScore from 27.76 to 176.61. By cluster analysis, we found the structures mainly referring to isoflavone, flavone and their glycoside, lignan, dihydrochalcone, polyphenol, catechin, and triterpenoid. To investigate the binding affinity and modes of these compounds, we selected 12 compounds with high score and representative structure. They were genistein, daidzein, phloretin, ellagic acid, ursolic acid, EGCG, kaempferol, naringenin, diosmin, naringin, silibinin, and genistein 7-O- $\beta$-D-glucoside. Genistein and daidzein were selected as two isoflavones, while kaempferol and naringenin were flavones. Phloretin, silibinin, ellagic acid, EGCG ((-)-epigallocatechin-3-gallate), and ursolic acid were representative of dihydrochalcone, lignin, polyphenol, catechin, and pentacyclic triterpenoid, respectively. Naringin, diosmin, and genistein $7-\mathrm{O}-\beta$-D-glucoside belonged to glycoside. Their binding affinities and modes with $\mathrm{ER} \alpha$ were investigated as follows.

3.5. Binding Affinities and Modes. The binding affinities of 12 compounds were measured by green PolarScreen ER $\alpha$ Competitor Assay. Among these compounds, diosmin, naringin, silibinin, and genistein 7-O- $\beta$-D-glucoside showed no significant binding affinity with the value of $\mathrm{IC}_{50}$ beyond $10 \mu \mathrm{M}$. Table 4 summarizes $\mathrm{IC}_{50}$ values of the other 8 compounds tested. Genistein had the highest inhibitory activity with $\mathrm{IC}_{50}$ value of $29.38 \pm 6.13 \mathrm{nM}$. But when genistein formed glycoside, the binding ability would be decreased sharply. The $\mathrm{IC}_{50}$ value of daidzein was $107.62 \pm 9.38 \mathrm{nM}$, not better than genistein. The two flavone compounds, kaempferol and naringenin, showed moderate affinity with the values of $\mathrm{IC}_{50}$ of $316.67 \pm 14.33 \mathrm{nM}$ and $967.54 \pm 70.95 \mathrm{nM}$, respectively, which suggested that the affinity of isoflavone was better than that of flavone. The common amino acid residues for the four compounds binding to ER $\alpha$ included Arg394 and Glu353 via conventional hydrogen bond/attractive charge and Leu387 and Leu391 via pi-alkyl interaction (Figure 5). Arg394 and Glu353 were also the key amino acid residues for 4-hydroxytamoxifen interacting $\mathrm{ER} \alpha$. Although 
<smiles>Cc1ccc(O)cn1</smiles>

Score: 1.746<smiles></smiles>

Score: 1.735<smiles>C=[NH+]C</smiles>

Score: -5.413<smiles>Cc1ccc(C)[nH]1</smiles>

Score: -4.452<smiles>Cc1cc(O)ccn1</smiles>

Score: 1.744<smiles>CN(C)CCOc1ccncc1</smiles>

Score: 1.733<smiles>C[NH+](C)C</smiles>

Score: -4.930

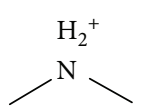

Score: -4.394<smiles>C=CC(O)=CC</smiles>

Score: 1.744<smiles>C=CC(=CC)OCCN(CC)CC</smiles>

Score: 1.733

(a)<smiles>Cc1ccc(O)cc1</smiles>

Score: 1.737

$\mathrm{HO}$<smiles>Oc1ccncc1</smiles>

Score: 1.737<smiles></smiles>

Score: 1.730

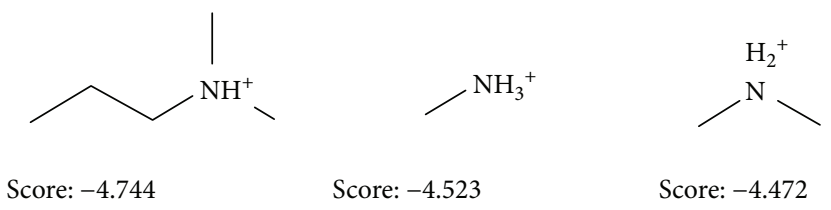

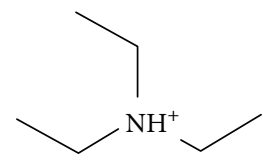

Score: -4.033<smiles></smiles>

Score: -4.005<smiles></smiles>

Score: -3.970

(b)

FIgURE 4: Examples of 10 good (a) and bad (b) fragments evaluated by the NB-b model. The Bayesian score (score) was given for each fragment.

TABLE 4: $\mathrm{IC}_{50}$ values (nM) of 8 representative compounds as $\mathrm{ER} \alpha$ antagonist from natural products and their binding affinity evaluated by Discovery Studio 2016.

\begin{tabular}{lccc}
\hline Chemical & $\mathrm{IC}_{50}(\mathrm{nM})$ & -CDOCKER_ENERGY & -CDOCKER_INTERACTION_ENERGY \\
\hline Estradiol & $7.38 \pm 0.80$ & 32.60 & 42.1106 \\
Genistein & $29.38 \pm 6.13$ & 31.63 & 45.9033 \\
Daidzein & $107.62 \pm 9.38$ & 38.321 & 42.813 \\
Phloretin & $74.55 \pm 24.24$ & 44.56 & 49.7287 \\
Ellagic acid & $62.61 \pm 9.34$ & 27.54 & 40.3217 \\
EGCG & $66.01 \pm 11.59$ & 46.72 & 64.262 \\
Ursolic acid & $977.38 \pm 125.30$ & -65.38 & 24.1527 \\
Kaempferol & $316.67 \pm 14.33$ & 30.31 & 41.5717 \\
Naringenin & $967.54 \pm 70.95$ & 30.08 & 38.021 \\
\hline
\end{tabular}




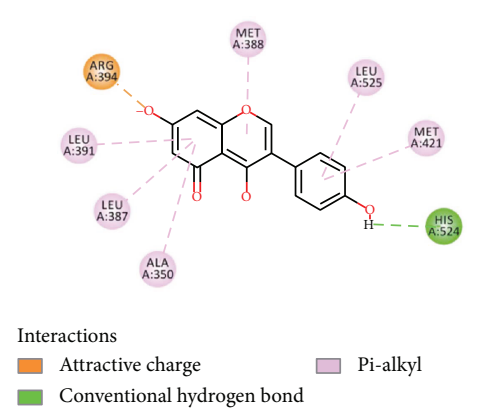

(a)

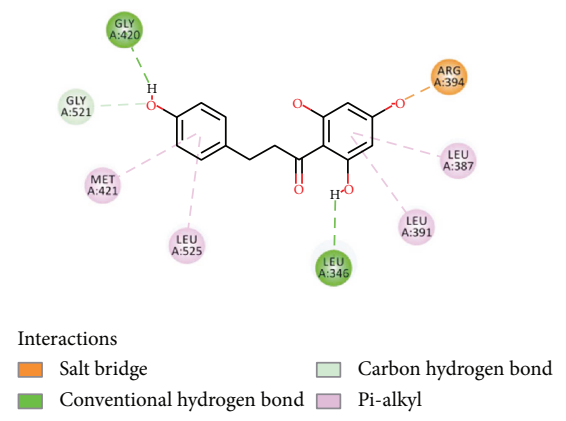

(d)

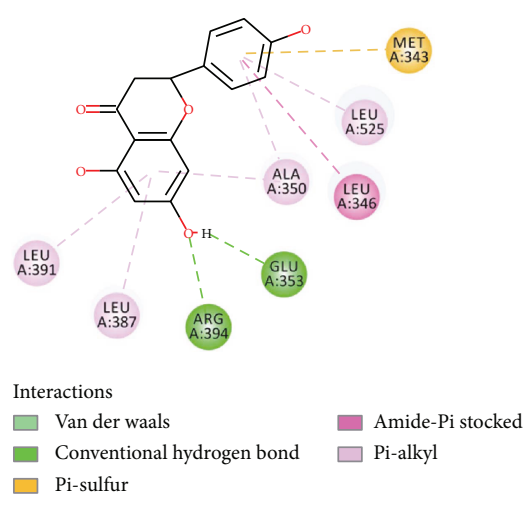

(b)

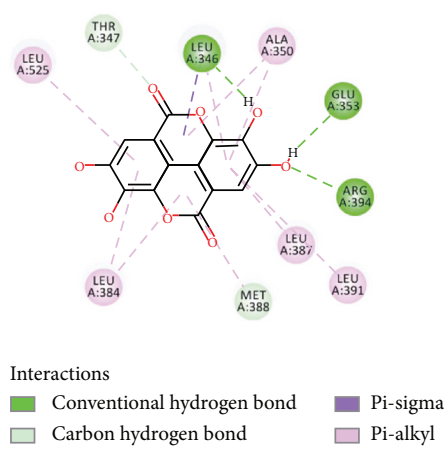

(e)

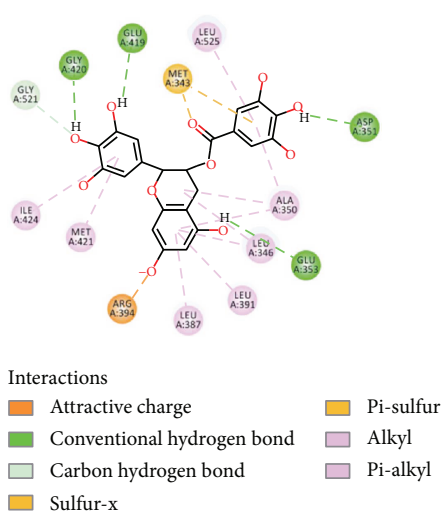

(c)

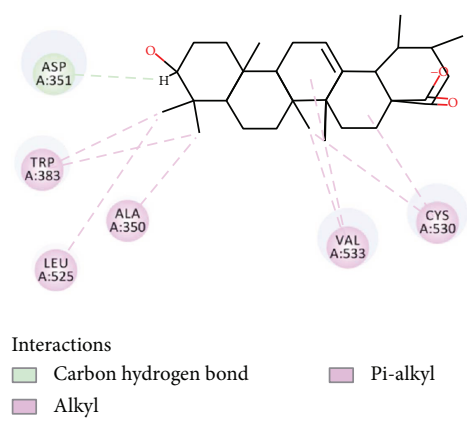

(f)

FIGURE 5: The investigation of the binding modes of 6 different skeleton structures. They were genistein (a), naringenin (b), EGCG (c), phloretin (d), ellagic acid (e), and ursolic acid (f), which belong to isoflavone, flavone, catechin, dihydrochalcone, polyphenol, and triterpenoid.

-CDOCKER energy and -CDOCKER interaction energy did not fluctuate significantly, the scores of isoflavones were a little better than those of flavones. In addition, compelling evidences suggested that isoflavones had multiple beneficial effects on breast and prostate cancers, menopausal symptoms, neurodegeneration, and so on [47]. Ellagic acid, phloretin, and EGCG did not belong to flavonoid skeleton, but they also had a better binding affinity. The values of $\mathrm{IC}_{50}$ were equally matched with $74.55 \pm 24.24 \mu \mathrm{M}, \quad 62.61 \pm 9.34 \mu \mathrm{M}$, and $66.01 \pm 11.59 \mu \mathrm{M}$, respectively. Polyphenol structures of EGCG and ellagic acid form a lot of hydrogen bonds, which lead to high binding affinity, but lack of hydroxyl group, ursolic acid had weak interaction with ER $\alpha$. One phenolic hydroxyl group of phloretin forms salt bridge with Arg394, which was different from other compounds (Figure 5). Therefore, phenolic hydroxyl groups and conjugated structures made these natural products high binding affinities, which were associated with the results of good fragments given by Bayesian model.

3.6. Antiestrogenic Effects. To explore whether 8 compounds have endocrine disrupting effects mediated by ER $\alpha$, we determined their antiestrogenic activities using luciferase reporter gene assay systems. Figure 6 showed their effects on the expression of ER $\alpha$. We found that genistein could decrease the expression of the ER $\alpha$ remarkably at a dose- dependent manner. Recent studies suggested genistein had an important role in the suppression of breast cancer via the competition of phytoestrogen with natural estrogens, declination of their bioavailability, and inhibition of cancer cell growth [48]. It was also reported that genistein can significantly attenuate oxidative stress by modulating the JNK3-mediated apoptosis, ERK1/2-mediated autophagy, and $\mathrm{TNF} \alpha$-associated inflammatory pathways [49]. Although daidzein was also attributed to isoflavone, the antagonist activity was lower than that of genistein. Ellagic acid, a plant-derived polyphenol, could also decrease the expression of ER $\alpha$ at high and medium concentrations. It was also reported that ellagic acid had an influence on ER $\alpha$-mediated signaling pathway in many kinds of cancer cell [50]. We provided the direct proof that ellagic acid had a strong interaction with $\mathrm{ER} \alpha$ and inhibited its expression. Phloretin is a natural dihydrochalcone and displays antioxidative and anti-inflammatory activity [51]. We firstly reported that phloretin could bind to ER $\alpha$ directly and regulated the expression of ER $\alpha$ in MCF-7 cells in a dose-dependent way. EGCG is one of the most potent and the most studied green tea catechins. There was much evidence about the involvement of EGCG for antioxidant and anti-inflammatory effects. Our results suggested that ER $\alpha$ was a target of EGCG to exhibit multiple pharmacological activities. Ursolic acid generated antiestrogenic effects only at high dose. Kaempferol and 


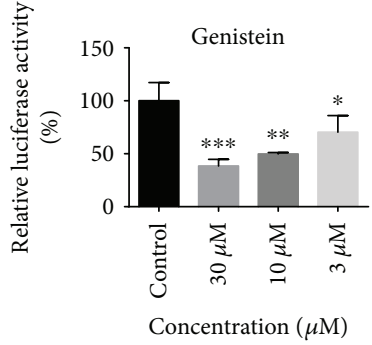

(a)

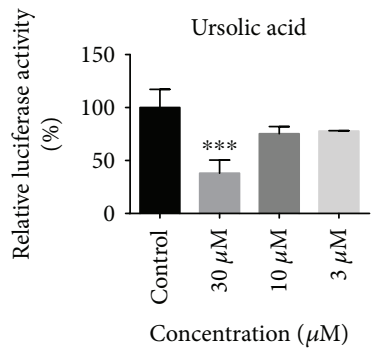

(e)

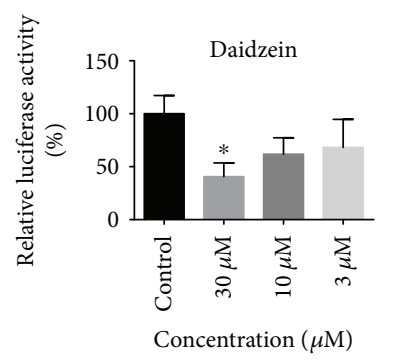

(b)

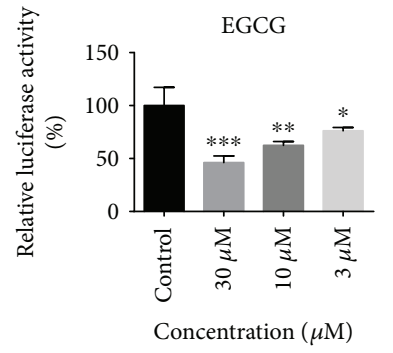

(f)

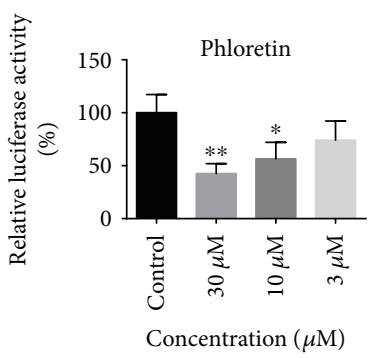

(c)

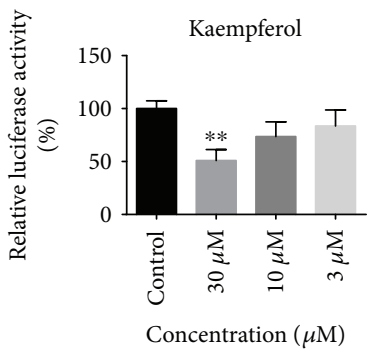

(g)

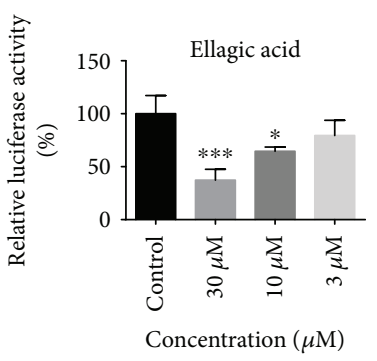

(d)

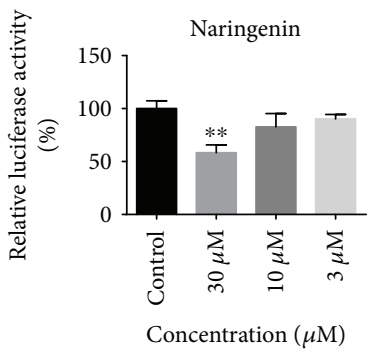

(h)

FIGURE 6: Antiestrogenic effects of 8 natural products in the ER $\alpha$ transactivation assay using MCF-7 cells transiently transfected with pERETATA-Luc. Cells were treated with the tested chemicals at a series of concentrations. The values represent the mean \pm SD of three independent experiments and are presented as the percentage of the response, with the control defined as $100 \%$. ${ }^{*} P<0.01,{ }^{* *} P<0.05$, and ${ }^{* * *} P<0.001$.

naringenin, as two flavone compounds, showed weak antiestrogenic effects, which was associated with the binding affinity results. It proved again that the antagonistic activity against ER $\alpha$ of isoflavone was superior to that of flavone.

\section{Conclusion}

In our study, we integrate the ligand- and structure-based methods for identification of $\operatorname{ER} \alpha$ antagonist from inhouse natural product library for the first time. As a result, 162 compounds were predicted as ER antagonists by NB-b and RP-d models, which were further evaluated by molecular docking, and 12 compounds were selected for activity validation. Based on the ER $\alpha$ competitor assay and luciferase reporter gene assay, we found 8 compounds exhibited antagonistic activity against $\mathrm{ER} \alpha$, including genistein, daidzein, phloretin, ellagic acid, ursolic acid, EGCG, kaempferol, and naringenin. The affinity of isoflavone was superior to flavone, and genistein had the highest inhibitory activity. However, the binding ability of genistein would be decreased significantly when it formed glycoside. It was also first reported that ellagic acid, phloretin, and EGCG could directly bind to the active pocket of $\mathrm{ER} \alpha$ with high affinity due to their phenolic hydroxyl group and conjugated structure. Therefore, natural products offer a rich resource for $\mathrm{ER} \alpha$ antagonist. In addition, virtual screening method for hit discovery and lead optimization would accelerate new drug discovery and increase efficiency and decrease the cost of the drug development process, contributing to more effective, safe drugs entering into market much at a quick pace.

\section{Data Availability}

The data used to support the findings of this study are available from the corresponding author upon request.

\section{Disclosure}

Xiaocong Pang and Weiqi Fu are the coauthors.

\section{Conflicts of Interest}

The authors declare no conflict of interest associated with this manuscript.

\section{Acknowledgments}

This research work was financially supported through grants from the National Great Science and Technology Projects (nos. 2013ZX09402203 and 2014ZX09507003002), CAMS Innovation Fund for Medical Sciences (CIFMS) (no. 2016I2M-3-007), and National Population and Health Scientific Data Sharing Platform (nos. 2016-NCMI-ZX-05 and NCMI-AGE05-201609).

\section{References}

[1] S. Nilsson and J.-Å. Gustafsson, "Estrogen receptors: therapies targeted to receptor subtypes," Clinical Pharmacology \& Therapeutics, vol. 89, no. 1, pp. 44-55, 2011.

[2] J. M. Hall, J. F. Couse, and K. S. Korach, "The multifaceted mechanisms of estradiol and estrogen receptor signaling," Journal of Biological Chemistry, vol. 276, no. 40, pp. 3686936872, 2001. 
[3] Y. Ishihara, K. Itoh, A. Ishida, and T. Yamazaki, "Selective estrogen-receptor modulators suppress microglial activation and neuronal cell death via an estrogen receptor-dependent pathway," The Journal of Steroid Biochemistry and Molecular Biology, vol. 145, pp. 85-93, 2015.

[4] P. A. Arias-Loza, M. Muehlfelder, and T. Pelzer, "Estrogen and estrogen receptors in cardiovascular oxidative stress," Pflügers Archiv - European Journal of Physiology, vol. 465, no. 5, pp. 739-746, 2013.

[5] I. Paterni, C. Granchi, J. A. Katzenellenbogen, and F. Minutolo, "Estrogen receptors alpha $(\mathrm{ER} \alpha)$ and beta $(\mathrm{ER} \beta)$ : subtype-selective ligands and clinical potential," Steroids, vol. 90, pp. 13-29, 2014.

[6] L. Zhang, P. Liu, H. Chen et al., "Characterization of a selective inverse agonist for estrogen related receptor $\alpha$ as a potential agent for breast cancer," European Journal of Pharmacology, vol. 789, pp. 439-448, 2016.

[7] B. B. Busch, W. C. Stevens, R. Martin et al., "Identification of a selective inverse agonist for the orphan nuclear receptor estrogen-related receptor $\alpha$," Journal of Medicinal Chemistry, vol. 47, no. 23, pp. 5593-5596, 2004.

[8] W. F. Anderson, N. Chatterjee, W. B. Ershler, and O. W. Brawley, "Estrogen receptor breast cancer phenotypes in the surveillance, epidemiology, and end results database," Breast Cancer Research and Treatment, vol. 76, no. 1, pp. 27-36, 2002.

[9] P. Ascenzi, A. Bocedi, and M. Marino, "Structure-function relationship of estrogen receptor $\alpha$ and $\beta$ : impact on human health," Molecular Aspects of Medicine, vol. 27, no. 4, pp. 299-402, 2006.

[10] H. S. Ranhotra, "Estrogen-related receptor alpha and cancer: axis of evil," Journal of Receptor and Signal Transduction Research, vol. 35, no. 6, pp. 505-508, 2015.

[11] F. E. May, "Novel drugs that target the estrogen-related receptor alpha: their therapeutic potential in breast cancer," Cancer Management and Research, vol. 6, pp. 225-252, 2014.

[12] R. A. Stein and D. P. McDonnell, "Estrogen-related receptor $\alpha$ as a therapeutic target in cancer," Endocrine-Related Cancer, vol. 13, Supplement 1, pp. S25-S32, 2006.

[13] Y. Shang and M. Brown, "Molecular determinants for the tissue specificity of SERMs," Science, vol. 295, no. 5564, pp. 2465-2468, 2002.

[14] V. C. Jordan, "Chemoprevention of breast cancer with selective oestrogen-receptor modulators," Nature Reviews Cancer, vol. 7, no. 1, pp. 46-53, 2007.

[15] F. E. van Leeuwen, A. W. van den Belt-Dusebout, F. E. van Leeuwen et al., "Risk of endometrial cancer after tamoxifen treatment of breast cancer," The Lancet, vol. 343, no. 8895, pp. 448-452, 1994.

[16] J. Shou, S. Massarweh, C. K. Osborne et al., "Mechanisms of tamoxifen resistance: increased estrogen receptor-HER2/neu cross-talk in ER/HER2-positive breast cancer," Journal of the National Cancer Institute, vol. 96, no. 12, pp. 926-935, 2004.

[17] T. Wang, Q. You, F. S. Huang, and H. Xiang, "Recent advances in selective estrogen receptor modulators for breast cancer," Mini-Reviews in Medicinal Chemistry, vol. 9, no. 10, pp. 1191-1201, 2009.

[18] D. D. Baker, M. Chu, U. Oza, and V. Rajgarhia, "The value of natural products to future pharmaceutical discovery," Natural Product Reports, vol. 24, no. 6, pp. 1225-1244, 2007.
[19] C. Y.-C. Chen, “TCM Database@Taiwan: the world’s largest traditional Chinese medicine database for drug screening in silico," PLoS One, vol. 6, no. 1, article e15939, 2011.

[20] J. Ru, P. Li, J. Wang et al., "TCMSP: a database of systems pharmacology for drug discovery from herbal medicines," Journal of Cheminformatics, vol. 6, no. 1, p. 13, 2014.

[21] R. Xue, Z. Fang, M. Zhang, Z. Yi, C. Wen, and T. Shi, “TCMID: Traditional Chinese Medicine integrative database for herb molecular mechanism analysis," Nucleic Acids Research, vol. 41, no. D1, pp. D1089-D1095, 2013.

[22] J. Huang and J. H. Wang, "CEMTDD: Chinese Ethnic Minority Traditional Drug Database," Apoptosis, vol. 19, no. 9, pp. 1419-1420, 2014.

[23] U. Schmidt, S. Struck, B. Gruening et al., "SuperToxic: a comprehensive database of toxic compounds," Nucleic Acids Research, vol. 37, Supplement 1, pp. D295-D299, 2009.

[24] M. Dunkel, M. Fullbeck, S. Neumann, and R. Preissner, "SuperNatural: a searchable database of available natural compounds," Nucleic Acids Research, vol. 34, Supplement 1, pp. D678-D683, 2006.

[25] T. Xie, S. Song, S. Li, L. Ouyang, L. Xia, and J. Huang, "Review of natural product databases," Cell Proliferation, vol. 48, no. 4, pp. 398-404, 2015.

[26] H. H. Xiao, C. Y. Fung, S. K. Mok et al., "Flavonoids from Herba epimedii selectively activate estrogen receptor alpha $(\mathrm{ER} \alpha)$ and stimulate ER-dependent osteoblastic functions in UMR-106 cells," The Journal of Steroid Biochemistry and Molecular Biology, vol. 143, pp. 141-151, 2014.

[27] W. L. Jorgensen, "The many roles of computation in drug discovery," Science, vol. 303, no. 5665, pp. 1813-1818, 2004.

[28] P. S. Kharkar, M. E. A. Reith, and A. K. Dutta, “Three-dimensional quantitative structure-activity relationship (3D QSAR) and pharmacophore elucidation of tetrahydropyran derivatives as serotonin and norepinephrine transporter inhibitors," Journal of Computer-Aided Molecular Design, vol. 22, no. 1, pp. 1-17, 2008.

[29] A. N. Lima, E. A. Philot, G. H. G. Trossini, L. P. B. Scott, V. G. Maltarollo, and K. M. Honorio, "Use of machine learning approaches for novel drug discovery," Expert Opinion on Drug Discovery, vol. 11, no. 3, pp. 225-239, 2016.

[30] A. Lavecchia, "Machine-learning approaches in drug discovery: methods and applications," Drug Discovery Today, vol. 20, no. 3, pp. 318-331, 2015.

[31] T. Liu, Y. Lin, X. Wen, R. N. Jorissen, and M. K. Gilson, "BindingDB: a web-accessible database of experimentally determined protein-ligand binding affinities," Nucleic Acids Research, vol. 35, Supplement 1, pp. D198-D201, 2007.

[32] M. M. Mysinger, M. Carchia, J. J. Irwin, and B. K. Shoichet, "Directory of useful decoys, enhanced (DUD-E): better ligands and decoys for better benchmarking," Journal of Medicinal Chemistry, vol. 55, no. 14, pp. 6582-6594, 2012.

[33] J. Fang, R. Yang, L. Gao et al., "Predictions of BuChE inhibitors using support vector machine and naive Bayesian classification techniques in drug discovery," Journal of Chemical Information and Modeling, vol. 53, no. 11, pp. 3009-3020, 2013.

[34] Y. Kong, D. Qu, X. Chen, Y. N. Gong, and A. Yan, "Selforganizing map (SOM) and support vector machine (SVM) models for the prediction of human epidermal growth factor receptor (EGFR/ ErbB-1) inhibitors," Combinatorial Chemistry \& High Throughput Screening, vol. 19, no. 5, pp. 400-411, 2016. 
[35] X. Xia, E. G. Maliski, P. Gallant, and D. Rogers, "Classification of kinase inhibitors using a Bayesian model," Journal of Medicinal Chemistry, vol. 47, no. 18, pp. 4463-4470, 2004.

[36] L. Chen, Y. Li, Q. Zhao, H. Peng, and T. Hou, “ADME evaluation in drug discovery. 10. Predictions of P-glycoprotein inhibitors using recursive partitioning and naive Bayesian classification techniques," Molecular Pharmaceutics, vol. 8, no. 3, pp. 889-900, 2011.

[37] A. K. Shiau, D. Barstad, P. M. Loria et al., "The structural basis of estrogen receptor/coactivator recognition and the antagonism of this interaction by tamoxifen," Cell, vol. 95, no. 7, pp. 927-937, 1998.

[38] J. M. M. Salmani, H. Lv, S. Asghar, and J. Zhou, "Amorphous solid dispersion with increased gastric solubility in tandem with oral disintegrating tablets: a successful approach to improve the bioavailability of atorvastatin," Pharmaceutical Development and Technology, vol. 20, no. 4, pp. 465-472, 2015.

[39] X. Pang, L. Wang, D. Kang et al., "Effects of P-glycoprotein on the transport of DL0410, a potential multifunctional antiAlzheimer agent," Molecules, vol. 22, no. 8, p. 1246, 2017.

[40] X. Pang, H. Fu, S. Yang et al., "Evaluation of novel dual acetyl- and butyrylcholinesterase inhibitors as potential anti-Alzheimer's disease agents using pharmacophore, 3DQSAR, and molecular docking approaches," Molecules, vol. 22, no. 8, p. 1254, 2017.

[41] S. P. Niinivehmas, E. Manivannan, S. Rauhamäki, J. Huuskonen, and O. T. Pentikäinen, "Identification of estrogen receptor $\alpha$ ligands with virtual screening techniques," Journal of Molecular Graphics and Modelling, vol. 64, pp. 30-39, 2016.

[42] Q. Zhang, M. Lu, C. Wang, J. Du, P. Zhou, and M. Zhao, "Characterization of estrogen receptor $\alpha$ activities in polychlorinated biphenyls by in vitro dual-luciferase reporter gene assay," Environmental Pollution, vol. 189, pp. 169-175, 2014.

[43] F. Cheng, W. Li, Y. Zhou et al., "admetSAR: a comprehensive source and free tool for assessment of chemical ADMET properties," Journal of Chemical Information and Modeling, vol. 52, no. 11, pp. 3099-3105, 2012.

[44] Z. Tang, C. Wu, T. Wang et al., "Design, synthesis and evaluation of 6-aryl-indenoisoquinolone derivatives dual targeting ER $\alpha$ and VEGFR-2 as anti-breast cancer agents," European Journal of Medicinal Chemistry, vol. 118, pp. 328-339, 2016.

[45] K. Ohta, Y. Chiba, A. Kaise, and Y. Endo, "Structure-activity relationship study of diphenylamine-based estrogen receptor (ER) antagonists," Bioorganic \& Medicinal Chemistry, vol. 23, no. 4, pp. 861-867, 2015.

[46] C. Li, C. Tang, Z. Hu et al., "Synthesis and structure-activity relationships of novel hybrid ferrocenyl compounds based on a bicyclic core skeleton for breast cancer therapy," Bioorganic \& Medicinal Chemistry, vol. 24, no. 13, pp. 3062-3074, 2016.

[47] C. Nagata, "Factors to consider in the association between soy isoflavone intake and breast cancer risk," Journal of Epidemiology, vol. 20, no. 2, pp. 83-89, 2010.

[48] A. Sureda, A. Sanches Silva, D. I. Sánchez-Machado et al., "Hypotensive effects of genistein: from chemistry to medicine," Chemico-Biological Interactions, vol. 268, pp. 37-46, 2017.

[49] S. Saha, P. Sadhukhan, S. Mahalanobish, S. Dutta, and P. C. Sil, "Ameliorative role of genistein against age-dependent chronic arsenic toxicity in murine brains via the regulation of oxidative stress and inflammatory signaling cascades," The Journal of Nutritional Biochemistry, vol. 55, pp. 26-40, 2017.
[50] Z. Papoutsi, E. Kassi, A. Tsiapara, N. Fokialakis, G. P. Chrousos, and P. Moutsatsou, "Evaluation of estrogenic/antiestrogenic activity of ellagic acid via the estrogen receptor subtypes ER $\alpha$ and ER $\beta$," Journal of Agricultural and Food Chemistry, vol. 53, no. 20, pp. 7715-7720, 2005.

[51] E. J. Lee, J. L. Kim, Y. H. Kim, M. K. Kang, J. H. Gong, and Y. H. Kang, "Phloretin promotes osteoclast apoptosis in murine macrophages and inhibits estrogen deficiencyinduced osteoporosis in mice," Phytomedicine, vol. 21, no. 10, pp. 1208-1215, 2014. 


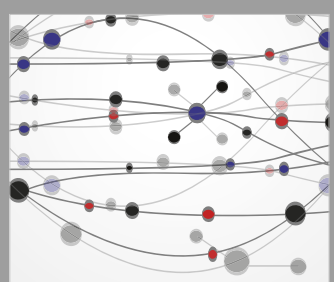

The Scientific World Journal
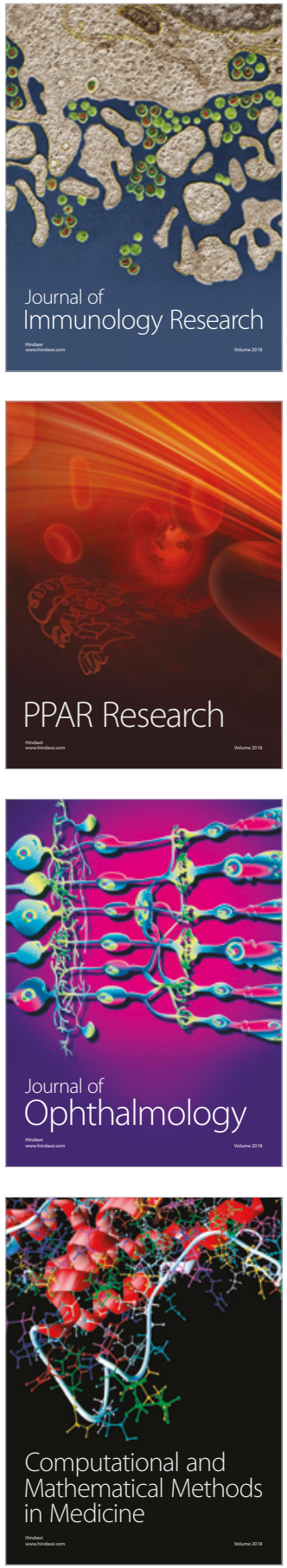

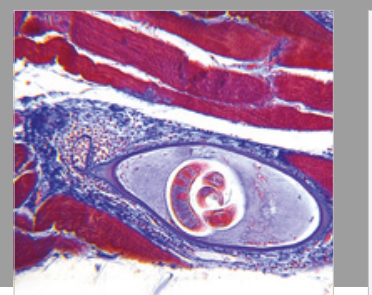

Gastroenterology Research and Practice

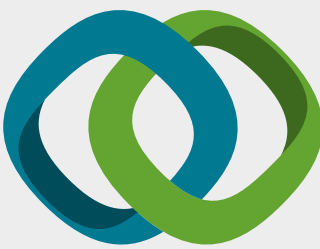

\section{Hindawi}

Submit your manuscripts at

www.hindawi.com
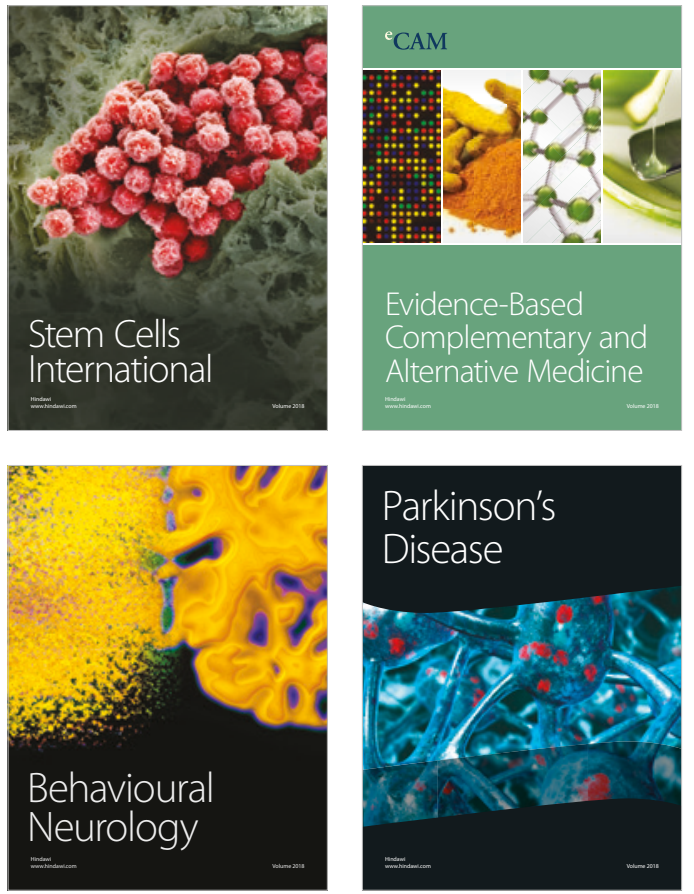

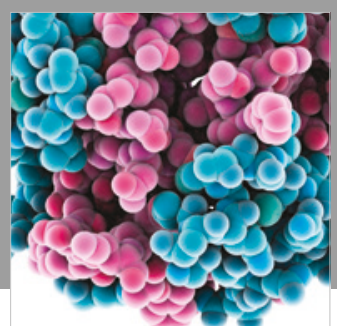

ournal of

Diabetes Research

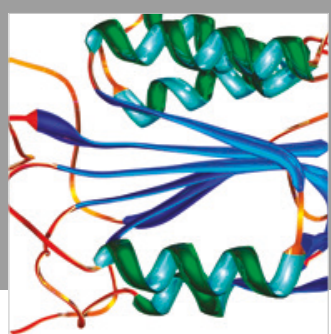

Disease Markers
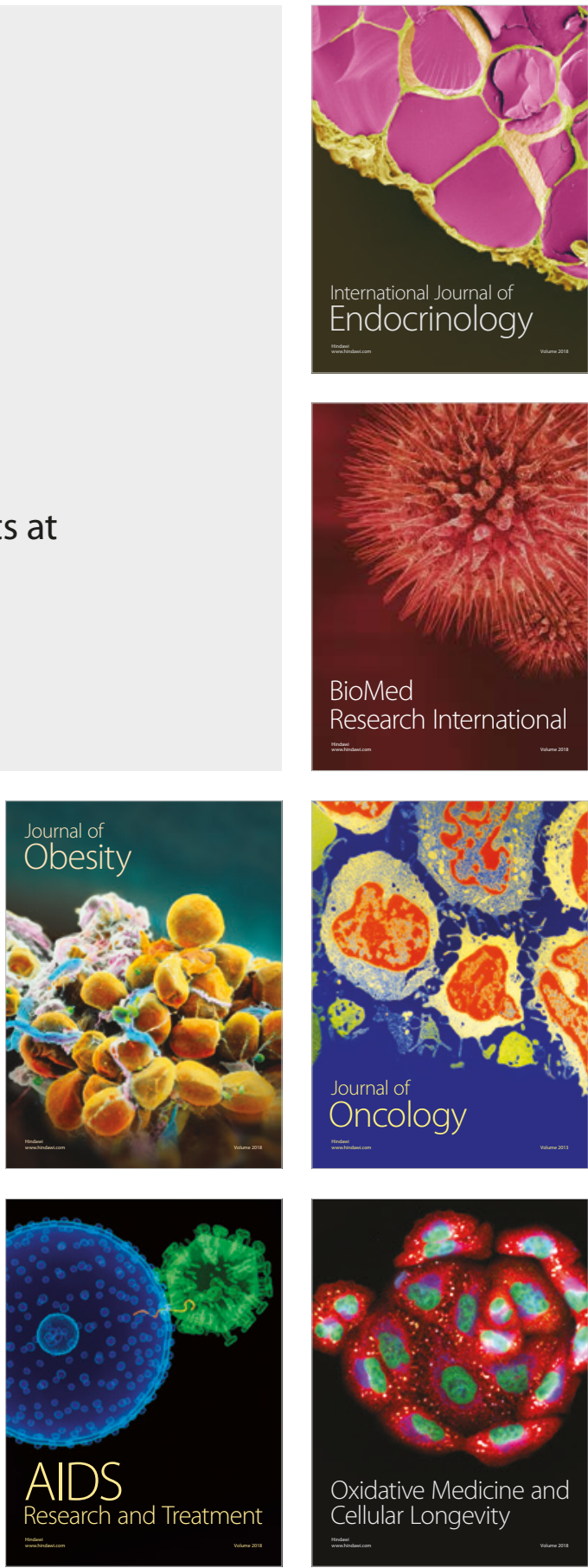